Impact of FAB classification on predicting outcome in acute myeloid leukemia, not otherwise specified, patients undergoing allogeneic stem cell transplantation in CR1 : An analysis of 1690 patients from the acute leukemia working party of EBMT

Canaani, Jonathan

2017-04

Canaani , J , Beohou , E , Labopin , M , Socie , G , Huynh , A, Volin , L , Cornelissen , J , Milpied, N, Gedde-Dahl , T, Deconinck, E, Fegueux, N, Blaise , D, Mohty , M \& Nagler , A 2017 , ' Impact of FAB classification on predicting outcome in acute myeloid leukemia, not otherwise specified, patients undergoing allogeneic stem cell transplantation in CR1 : An analysis of 1690 patients from the acute leukemia working party of EBMT ' , American Journal of Hematology, vol. 92 , no. 4 , pp. 344-350 . https://doi.org/10.1002/ajh.24640

http://hdl.handle.net/10138/206990

https://doi.org/10.1002/ajh.24640

unspecified

publishedVersion

Downloaded from Helda, University of Helsinki institutional repository.

This is an electronic reprint of the original article.

This reprint may differ from the original in pagination and typographic detail.

Please cite the original version. 


\title{
Impact of FAB classification on predicting outcome in acute myeloid leukemia, not otherwise specified, patients undergoing allogeneic stem cell transplantation in CR1: An analysis of 1690 patients from the acute leukemia working party of EBMT
}

\author{
Jonathan Canaani ${ }^{1}$ (1) | Eric Beohou 2,3 | Myriam Labopin ${ }^{2,3}$ | Gerard Socié ${ }^{3}$ | \\ Anne Huynh $^{4}$ | Liisa Volin ${ }^{5}$ | Jan Cornelissen ${ }^{6}$ | Noel Milpied7 | \\ Tobias Gedde-Dahl $^{8}$ | Eric Deconinck ${ }^{9}$ | Nathalie Fegueux ${ }^{10}$ | Didier Blaise ${ }^{11}$ | \\ Mohamad Mohty ${ }^{2}$ | Arnon Nagler 1,3
}

\author{
${ }^{1}$ Hematology Division, Chaim Sheba Medical \\ Center, Tel Hashomer, Tel Aviv University, \\ Israel; ${ }^{2}$ Hématologie Clinique et Thérapie \\ Cellulaire, Hôpital Saint-Antoine, Universite \\ Pierre \& Marie Curie, Paris, France; ${ }^{3}$ EBMT \\ Acute Leukemia Working Party office, \\ Hôpital Saint-Antoine, Paris, France; ${ }^{4}$ Institut \\ Universitaire du Cancer Toulouse, Oncopole, \\ Toulouse, France; ${ }^{5} \mathrm{HUCH}$ Comprehensive \\ Cancer Center, Stem Cell Transplantation \\ Unit, Helsinki, Finland; ' $D$ Department of \\ Hematology, Erasmus MC Cancer Institute, \\ University Medical Center Rotterdam, \\ Rotterdam, The Netherlands; ${ }^{7} \mathrm{CHU}$ \\ Bordeaux, Hôpital Haut-leveque, Pessac, \\ France; ${ }^{8}$ Department of Hematology, Clinic \\ for Cancer, Surgery and Transplantation, \\ Oslo University Hospital, Rikshospitalet, \\ Oslo, Norway; ${ }^{9}$ Service dHématologie, \\ Hopital Jean Minjoz, Besancon, France; \\ ${ }^{10}$ Département dHématologie Clinique, $\mathrm{CHU}$ \\ Lapeyronie, Montpellier, France; ${ }^{11}$ Centre de \\ Recherche en Cancérologie de Marseille, \\ Programme de Transplantation \& Therapie \\ Cellulaire, Marseille, France

\section{Correspondence} \\ Arnon Nagler, Division of Hematology, \\ Chaim Sheba Medical Center, Tel- \\ Hashomer, Ramat-Gan 52621, Israel. \\ Email: arnon.nagler@sheba.health.gov.il
}

\begin{abstract}
The French, American, and British (FAB) classification system for acute myeloid leukemia ( $A M L$ ) is extensively used and is incorporated into the AML, not otherwise specified (NOS) category in the 2016 WHO edition of myeloid neoplasm classification. While recent data proposes that FAB classification does not provide additional prognostic information for patients for whom NPM1 status is available, it is unknown whether $F A B$ still retains a current prognostic role in predicting outcome of $A M L$ patients undergoing allogeneic stem cell transplantation. Using the European Society of Blood and Bone Marrow Transplantation registry we analyzed outcome of 1690 patients transplanted in $\mathrm{CR} 1$ to determine if $\mathrm{FAB}$ classification provides additional prognostic value. Multivariate analysis revealed that M6/M7 patients had decreased leukemia free survival (hazard ratio (HR) of $1.41,95 \%$ confidence interval $(\mathrm{Cl}), 1.01-1.99 ; P=.046)$ in addition to increased nonrelapse mortality (NRM) rates (HR, 1.79; 95\% Cl, 1.06-3.01; $P=.028)$ compared with other $F A B$ types. In the $N P M 1^{w t}$ AML, NOS cohort, FAB M6/M7 was also associated with increased NRM (HR, 2.17; 95\% $\mathrm{Cl}, 1.14-4.16 ; P=.019)$. Finally, in FLT3-ITD ${ }^{+}$patients, multivariate analyses revealed that specific FAB types were tightly associated with adverse outcome. In conclusion, FAB classification may predict outcome following transplantation in AML, NOS patients.
\end{abstract}

\section{1 | INTRODUCTION}

Prognostication plays a major role in clinical decision-making for patients with acute myeloid leukemia (AML). With the advent of widespread high throughput molecular sequencing platforms, assessment of FLT3 and NPM1 mutational status has become a standard major determinant in predicting patient outcome. As ASCT is associated with sig- nificant rates of attendant toxicity and mortality, ${ }^{1}$ efforts to better prognosticate and risk stratify patients before transplant are of prime importance. Previous efforts aiming at delineating the role FLT3-ITD status $^{2-8}$ and NPM1 status ${ }^{9-11}$ play in determining clinical outcome following ASCT have yielded conflicting results, thus underscoring the need for improved prognostication of candidates for ASCT. The French-American-British (FAB) classification system has been 
traditionally used to classify AML into discrete types designated M0M7, and notwithstanding the incorporation of new molecular categories into the recent 2016 rendition of the world health organization (WHO) myeloid neoplasms classification, ${ }^{12}$ FAB classification is still a core feature of the large WHO category of AML, not otherwise specified (NOS) patients. While earlier studies suggested a prognostic role for specific $F A B$ types, ${ }^{13-17}$ the prognostic role $F A B$ classification holds in current practice has been recently questioned by Walter et al. who propose that when NPM1 and CEBPA status is available, FAB no longer predicts patient outcome. ${ }^{18}$ Yet, it remains unclear whether in patients undergoing allogeneic stem cell transplant (ASCT) in complete remission (CR1), FAB still holds prognostic significance. To this end, using the European Society of Blood and Bone Marrow Transplantation (EBMT) registry, we set out to determine whether $F A B$ classification still retains a significant role in the current era of advanced molecularly incorporated classification systems of AML.

\section{2 | PATIENTS AND METHODS}

\subsection{Study population}

Data were provided, and the study design was approved by the acute leukemia working party (ALWP) of the EBMT group registry, in accordance with the EBMT guidelines for retrospective studies. EBMT is a voluntary working group of more than 500 transplant centers which are required to report all consecutive stem cell transplantations and follow-up once a year. Audits are routinely performed to determine the accuracy of the data. Using the EBMT registry we identified patients with available $F A B$ data and the following inclusion criteria: (1) age over 18, (2) de novo non-M3 AML, and (3) ASCT in complete remission (CR1) from a fully matched sibling or a 10/10 human leukocyte antigen (HLA) matched unrelated donor. Patients with recurrent genetic abnormalities, AML with myelodysplasia-related changes, and patients with therapy-related myeloid neoplasms were excluded from the analysis.

\section{2 | Endpoint definitions}

The primary end points were leukemia-free survival (LFS), relapse incidence (RI), nonrelapse mortality (NRM), and overall survival (OS). ISecondary end points were engraftment, acute and chronic graftversus-host disease (GVHD). LFS was defined as survival with no evidence of relapse or progression. Relapse was defined as the reappearance of $5 \%$ bone marrow blasts and/or extramedullary lesion due to specific blast cell infiltration. NRM was defined as death without evidence of relapse or progression. OS was defined as the time from ASCT to death, regardless of the cause.

\subsection{Statistical analysis}

Multivariate analyses were used to assess whether the FAB type was independently associated with LFS, OS, RI, and NRM. Age, gender, cytogenetics, donor type, FMS-like tyrosine kinase-3 internal tandem duplication (FLT3-ITD) status, nucleophosmin (NPM1) status, and conditioning intensity were covariates for regression modeling. Cytogenetic risk groups were defined according to established criteria. ${ }^{19}$ LFS was defined as time interval from ASCT until either relapse or death in months, and was calculated using the Kaplan-Meier estimate. NRM was defined as death in the absence of relapse. RI and NRM were calculated using cumulative incidence curves in a competing risks setting, death in remission being treated as a competing event to relapse and relapse in the NRM estimation setting, respectively. Univariate analyses were performed using log rank test for OS and LFS while Gray's test was applied for RI and NRM. A Cox proportional hazards model was used for multivariate regression. Variables differing in terms of distribution between the groups and factors conceptually important were included in the model. Results are expressed as hazard ratio (HR) with 95\% confidence interval (Cl). All tests were two-sided. The type I error rate was fixed at 0.05 for determination of factors associated with time to event outcomes.

Statistical analyses were performed with SPSS 19 (SPSS Inc., Chicago, USA) and R (R Development Core Team, Vienna, Austria) software packages.

\section{3 | RESULTS}

\section{1 | Patient characteristics}

A cohort of 1690 patients who were transplanted between 2005 and 2014 was identified and analyzed; baseline characteristics are shown in Table 1. The median age was 50 years (range, 18-74 years) with a median follow-up duration of 34.7 months. Patients with M6 and M7 were significantly older compared with patients with other FAB types. M7 patients also had an increased rate of poor risk cytogenetics (60\%) although we note the small number of patients in this category $(n=$ 10) may limit interpretation of this observation. Patients with $M 2$ and M4 had the highest rates of mutated FLT3-ITD (54.6\% and 53.6\%, respectively) while the group of M4 patients was noted to have the highest prevalence of NPM1 mutations (53\% versus $38.6 \%$ for the entire cohort). Donor type and conditioning intensity did not differ to a significant degree between the different FAB types.

\subsection{Impact of FAB category on clinical outcome in the complete cohort}

To assess the impact of FAB category on patient outcome we initially analyzed the cohort as a whole, namely irrespective of NPM1 and FLT3-ITD status. In univariate analysis there was no significant association of $F A B$ category with clinical outcome following transplant, specifically rates of LFS, OS, RI, and NRM were comparable between the different FAB subtypes (Supporting Information Table 1). Since M6 and M7 patients comprised together the smallest group of patients in our cohort ( $n=75$ ), a reanalysis comparing the composite of M6/M7 patients to M0-M5 patients was performed. In univariate analysis M6/ M7 patients had increased 1 year (15.6\% [8.2-25] versus 9.5\% [8.111.1]) and 3 years (24.1\% [13.9-35.7] versus $13.7 \%$ [11.9-15.6]) $(P=$ 0.0291) NRM rates compared with non-M6/M7 patients, while LFS, $\mathrm{OS}$, and RI did not differ to a significant degree between groups. To 


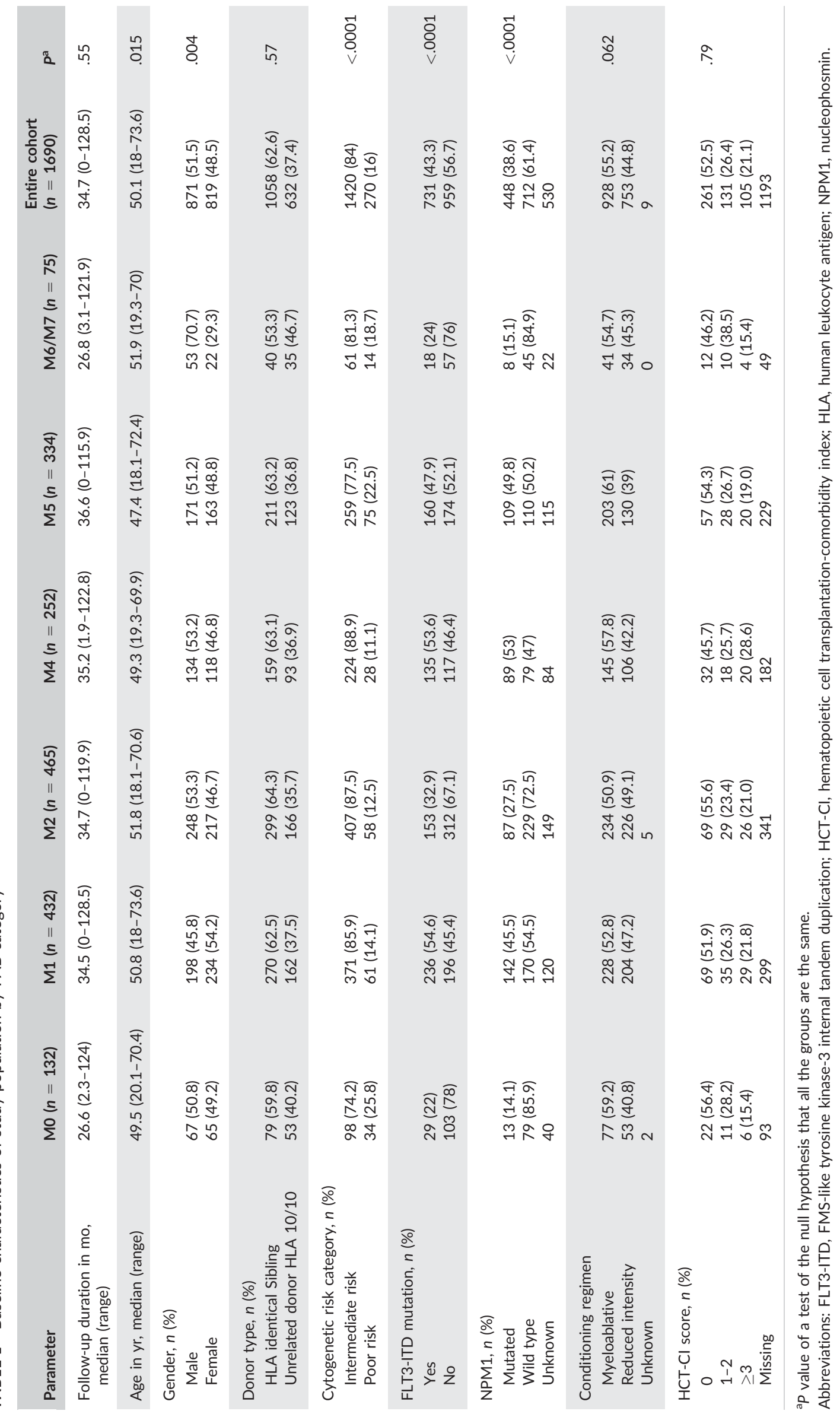


TABLE 2 Multivariable analysis of the entire cohort for clinical outcome

\begin{tabular}{|c|c|c|c|c|}
\hline Parameter & $\begin{array}{l}\text { Leukemia-free survival HR } \\
(95 \% \mathrm{Cl})\end{array}$ & $\begin{array}{l}\text { Overall survival } \\
\text { HR }(95 \% \mathrm{Cl})\end{array}$ & $\begin{array}{l}\text { Relapse incidence } \\
\text { HR }(95 \% \mathrm{Cl})\end{array}$ & $\begin{array}{l}\text { Nonrelapse mortality } \\
\text { HR }(95 \% \mathrm{CI})\end{array}$ \\
\hline $\begin{array}{l}\text { FAB classification (M6/M7 } \\
\text { us. non M6/M7) }\end{array}$ & 1.41 (1.01-1.99), $\mathrm{P}=.046$ & 1.28 (0.89-1.85), $\mathrm{P}=.19$ & $1.22(0.77-1.92), P=.39$ & $1.79(1.06-3.01), P=.028$ \\
\hline Female gender & 0.89 (0.76-1.04), $\mathrm{P}=.15$ & 0.89 (0.75-1.05), $P=.16$ & $0.86(0.71-1.04), P=.12$ & $0.97(0.74-1.26), P=.8$ \\
\hline Age (10-year increment) & $1.13(1.05-1.21), P=.001$ & 1.22 (1.13-1.32), $\mathrm{P}<.0001$ & $1.04(0.95-1.14), P=.4$ & 1.33 (1.17-1.52), $\mathrm{P}<.0001$ \\
\hline $\begin{array}{l}\text { Donor (unrelated } 10 / 10 \\
\text { vs. HLA identical sibling) }\end{array}$ & 1.00 (0.85-1.18), $P=.96$ & $1.07(0.9-1.27), P=.47$ & 0.87 (0.71-1.07), $P=.2$ & $1.3(0.99-1.71), P=.063$ \\
\hline $\begin{array}{l}\text { Cytogenetic risk status } \\
\text { (poor vs. intermediate) }\end{array}$ & 1.55 (1.26-1.89), $\mathrm{P}<.0001$ & 1.65 (1.34-2.04), $\mathrm{P}<.0001$ & 1.71 (1.34-2.18), $\mathrm{P}<.0001$ & $1.22(0.85-1.76), P=.27$ \\
\hline $\begin{array}{l}\text { FLT3-ITD status } \\
\text { (present vs. absent) }\end{array}$ & $0.74(0.58-0.93), P=.011$ & $0.72(0.56-0.92), P=.009$ & 0.77 (0.58-1.02), $P=.068$ & $0.69(0.46-1.04), P=.073$ \\
\hline $\begin{array}{l}\text { NPM1 status } \\
\text { (mutated vs. wild type) }\end{array}$ & $1.06(0.88-1.28), P=.54$ & $1.08(0.89-1.31), P=.45$ & $1.03(0.82-1.3), P=.78$ & $1.13(0.82-1.55), P=.45$ \\
\hline $\begin{array}{l}\text { Conditioning intensity } \\
\text { (RIC vs. MAC) }\end{array}$ & $1.01(0.84-1.21), P=.91$ & $1.00(0.82-1.21), P=.98$ & 1.18 (0.94-1.47), $P=.15$ & 0.74 (0.54-1.01), $P=.056$ \\
\hline
\end{tabular}

Abbreviations: FLT3-ITD, FMS-like tyrosine kinase-3 internal tandem duplication; MAC, myeloablative conditioning; NPM1, nucleophosmin; RIC, reduced intensity conditioning.

account for the possible influence of additional factors on the impact of FAB subtype on clinical outcome, a multivariate analysis was undertaken using the following covariates: age, gender, cytogenetic risk category, donor type, FLT3-ITD status, NPM1 status, and conditioning intensity. As shown in Table 2 and Figure 1, M6/M7 patients had decreased LFS and increased NRM rates $(H R=1.41$ [1.01-1.99], $P=.046$; and $H R=1.79$ [1.06-3.01], $P=.028$ respectively), although these did not translate into a change in overall survival.

\section{3 | Impact of FAB classification on clinical outcome in NPM1 ${ }^{\text {wt }}$ "AML, NOS" patients}

Next, to align our data with the recently introduced 2016 WHO AML, NOS classification which comprises AML patients who lack any of the following: recurrent genetic abnormalities, mutated NPM1, biallelic mutations of CEBPA, myelodysplasia-related changes or therapy related AML, we restricted our analysis to the NPM1 ${ }^{\text {wt }}$ subset of the cohort $(n=712)$. Patients with unknown NPM1 status were excluded from the analysis. The characteristics of this cohort are summarized in Supporting Information Table 2, notably FAB M7 and M5 patients were more likely to have poor risk cytogenetics $(57.1 \%$ and $35.5 \%$ respectively), and additionally M4 patients were more likely to be FLT3-ITD ${ }^{+} /$NPM1 $^{w t}$ (26.6\% vs. $16.9 \%$ for the entire cohort). Subsequently, a multivariate regression analysis was performed, and as summarized in Table 3 and Supporting Information Figure 1 showed that in AML, NOS NPM1 ${ }^{\text {wt }}$ patients, FAB M6/M7 category was significantly associated with increased NRM (HR $=2.17$ [1.14-4.16], $P=.019)$. We also note that on additional analysis, upon comparing M6/M7 to the other FAB types individually, we observed that M6/M7 compared with $\mathrm{MO}$ was significantly associated with decreased LFS (HR $=1.88$ [1.023.48], $P=.045)$, increased NRM (HR $=2.7$ [1.05-6.93], $P=.039)$, and a trend towards decreased OS (HR=1.87 [0.96-3.64], $P=.066)$.

\subsection{Association of FAB category with clinical outcome in FLT3-ITD ${ }^{+}$patients following ASCT}

Given the known association of FLT3-ITD ${ }^{+}$status with deleterious outcome in $A M L$ in general and post-transplant, ${ }^{7,8}$ we wondered whether FAB classification would have additional prognostic value in this specific patient population ( $n=356$ ). The characteristics of this cohort are summarized in Supporting Information Table 3.

Univariate analysis indicated that M4 patients had a significantly lower 1 year OS rate of $64 \%(95 \% \mathrm{Cl}$ [56-73], $P=.0345)$ and a lower 3-year OS rate of 49.1\% (95\% Cl [40.6-59.4], $P=.0345$ ).

As shown in Supporting Information Table 4 in which MO serves as the reference considered to have a hazard ratio of 1.0, a multivariate analysis confirmed that FAB types M2 and M4 were independently associated with decreased LFS rates whereas M4 and M6/M7 had lower OS rates, and M2 patients had an increased likelihood of RI. There was no statistically significant difference in outcome between M6/M7 patients and non M6/M7 patients in the FLT3-ITD ${ }^{+}$cohort (Supporting Information Figure 2).

\section{4 | DISCUSSION}

In the present analysis of $1690 \mathrm{AML}$ patients undergoing allogeneic stem cell transplantation in CR1, we present data supporting a contemporary role for $F A B$ classification in the prediction of posttransplantation outcome. Our findings indicate that in specific patient subsets; eg M6/M7 in an unselected cohort, M6/M7 in the strictly defined AML, NOS NPM1 ${ }^{\text {wt }}$ subset, and M6/M7 in FLT3-ITD ${ }^{+}$patients, $F A B$ classification has a substantial prognostic value in predicting adverse outcome following allogeneic transplantation.

Since its introduction in the 1970s, FAB classification has played a central role in classification and to some degree prognostication of 

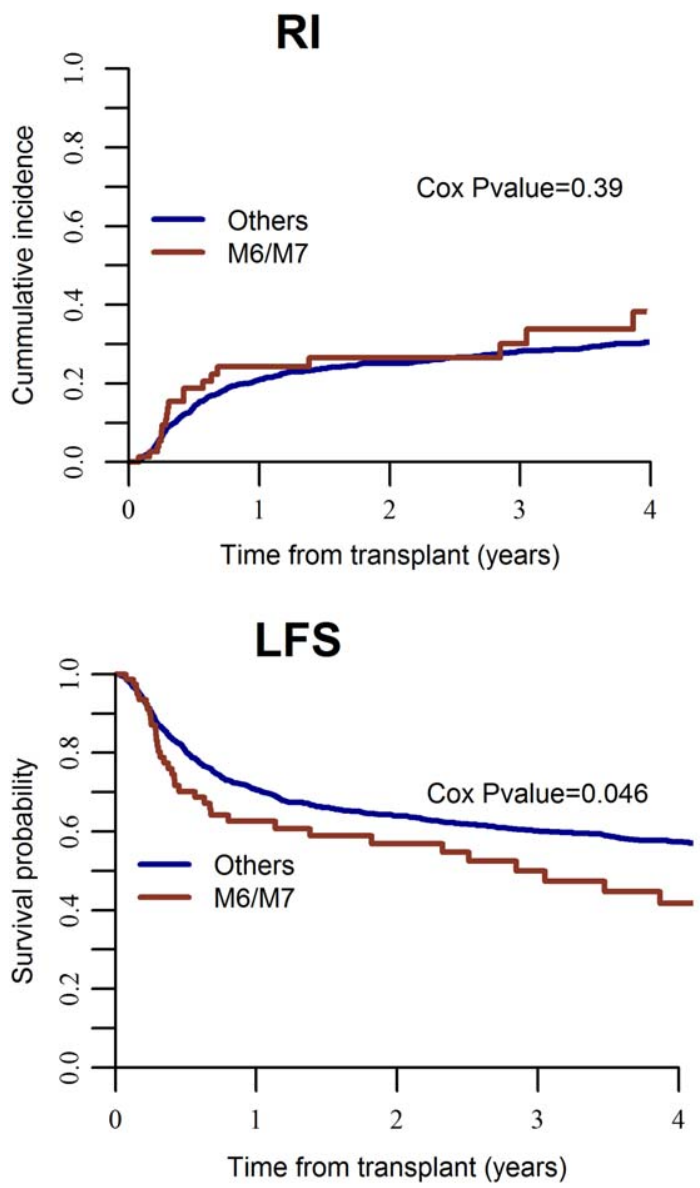
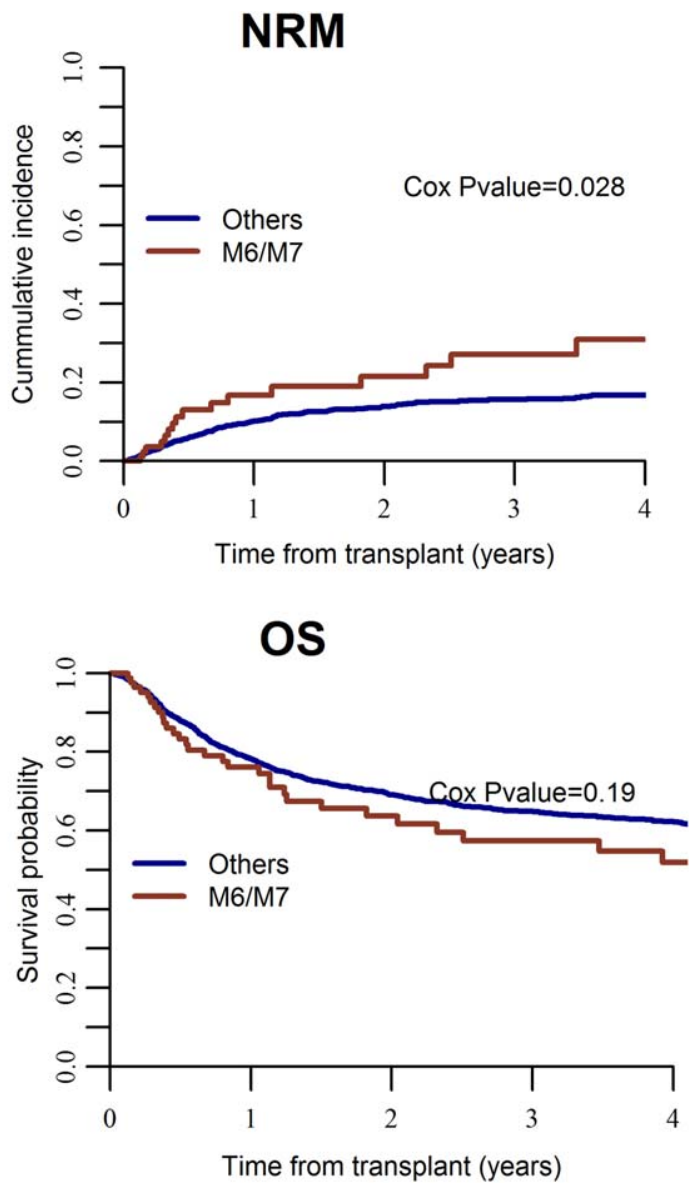

FIGURE 1 Outcome of FAB M6/M7 patients compared with other FAB types following ASCT. A, Relapse incidence (RI) after ASCT. B, Nonrelapse mortality (NRM). C, Leukemia-free survival (LFS). D, Overall survival (OS) after ASCT. Adjusted for age, gender, donor type, cytogenetic risk, FLT3-ITD status, NPM1 status, and conditioning regimen. [Color figure can be viewed at wileyonlinelibrary.com]

AML. $^{20-22}$ Subsequently it underwent modifications to accommodate for advances made in immunophenotyping, cytogenetics, and molecular genetics. ${ }^{23,24}$ While the most recent edition of the World Health Organization classification of myeloid neoplasms and acute leukemia, ${ }^{12}$ places emphasis on molecular categorization of $\mathrm{AML}$, eg, the addition of the two new categories of AML with mutated NPM1, and AML with biallelic mutations of CEBPA (and the provisional entities of AML with mutated RUNX1 or BCR-ABL1), FAB is still incorporated into the $A M L, N O S$ category which comprises most of the $A M L$ patient population.

TABLE 3 Multivariable analysis of AML, NOS NPM1 ${ }^{\text {wt }}$ for clinical outcome

\begin{tabular}{|c|c|c|c|c|}
\hline Parameter & $\begin{array}{l}\text { Leukemia-free survival } \\
\text { HR }(95 \% \mathrm{Cl})\end{array}$ & $\begin{array}{l}\text { Overall survival } \\
\text { HR }(95 \% \mathrm{CI})\end{array}$ & $\begin{array}{l}\text { Relapse incidence } \\
\text { HR (95\% CI) }\end{array}$ & $\begin{array}{l}\text { Nonrelapse mortality } \\
\text { HR }(95 \% \mathrm{CI})\end{array}$ \\
\hline $\begin{array}{l}\text { FAB classification } \\
\text { (M6/M7 vs. non-M6/M7) }\end{array}$ & $1.48(0.94-2.33), P=.093$ & $1.43(0.88-2.35), P=.15$ & $1.1(0.57-2.1), P=.78$ & $2.17(1.14-4.16), P=.019$ \\
\hline Female gender & $0.79(0.61-1.02), P=.071$ & $0.81(0.62-1.06), P=.12$ & $0.76(0.56-1.04), P=.088$ & $0.84(0.54-1.29), P=.43$ \\
\hline Age (10-year increment) & $1.15(1.02-1.29), P=.019$ & $1.25(1.1-1.42), P=.0007$ & $1.08(0.93-1.24), P=.31$ & $1.3(1.06-1.6), P=.012$ \\
\hline $\begin{array}{l}\text { Donor (unrelated 10/10 } \\
\text { vs. HLA identical sibling) }\end{array}$ & $1.08(0.83-1.39), P=.57$ & $1.17(0.89-1.53), P=.27$ & $0.92(0.66-1.26), P=.59$ & $1.42(0.93-2.16), P=.11$ \\
\hline $\begin{array}{l}\text { Cytogenetic risk status } \\
\text { (poor vs. intermediate) }\end{array}$ & $1.45(1.07-1.95), P=.015$ & $1.68(1.24-2.29), P=.0009$ & $1.83(1.28-2.6), P=.0009$ & $0.86(0.49-1.52), P=.61$ \\
\hline $\begin{array}{l}\text { FLT3-ITD status } \\
\text { (present vs. absent) }\end{array}$ & $1.96(1.45-2.65), P<.0001$ & $1.65(1.18-2.3), P=.003$ & 2.35 (1.64-3.36), $P<.0001$ & $1.33(0.77-2.31), P=.31$ \\
\hline $\begin{array}{l}\text { Conditioning intensity } \\
\text { (RIC vs. MAC) }\end{array}$ & $0.98(0.73-1.32), P=.91$ & $1.04(0.76-1.42), P=.82$ & $1.11(0.77-1.61), P=.58$ & $0.76(0.46-1.26), P=.29$ \\
\hline
\end{tabular}

Abbreviations: FLT3-ITD, FMS-like tyrosine kinase-3 internal tandem duplication; MAC, myeloablative conditioning; NPM1, nucleophosmin; RIC, reduced intensity conditioning; WT, wild type. 
In early studies looking at the prognostic role of FAB in the setting of allogeneic transplantation, M4 and M5 FAB subtypes were found to be associated with an increased relapse rate, $58 \%$ versus the $9 \%$ seen in M1-M3. ${ }^{15}$ In the same vein, registry data from EBMT also established FAB M4 and M5 as adverse risk factors predicting for leukemia relapse. ${ }^{16}$ Data published nearly a decade later showed that in a cohort of 91 patients undergoing transplant in CR1 from HLA-identical siblings, overall survival was inferior in M4-M6 FAB subtypes, and transplantrelated mortality was increased in FAB M4-M6 patients. ${ }^{17}$ More recent data in postautologous transplantation patients also supports a prognostic role for $F A B$ classification, wherein M0/M6/M7 patients experienced significantly decreased overall survival compared with M1-M5 patients. ${ }^{14}$ Our experience differs from that published by the European and Italian cooperative groups in the EORTC-GIMEMA AML 8A trial where 169 patients underwent ASCT in CR1 and where FAB type was not found to be of prognostic importance. ${ }^{25}$ However, we do note that given our much larger and more recent cohort, our data may reflect more accurately the typical contemporary transplant patient population. Additionally, the aforementioned study did not have molecular data which undoubtedly adds prognostic depth to our analysis.

Interestingly, most of the literature examining the role of FAB in predicting outcome of AML patients treated with chemotherapy only regimens or at least not analyzed specifically for transplant, does not indicate a prognostic role for FAB classification. Earlier published data from the Eastern Cooperative Oncology Group focusing on outcome of M5 versus non-M5 patients also did not support a prognostic role for FAB classification, although only seven patients (from a total of 81) underwent allogeneic transplantation in this analysis, thus limiting the ability to interpret data from a transplant standpoint. ${ }^{26}$ More recently, Walter et al. performed a comprehensive analysis of the combined datasets of several large cooperative groups in U.S. and Europe and determined that FAB MO was associated with inferior rates of attainment of a complete remission following induction chemotherapy resulting in poorer relapse-free and overall survival rates. Notably, upon restricting

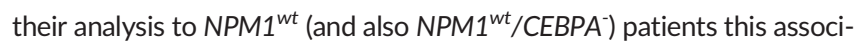
ation was no longer meaningful. ${ }^{18}$ These data differ to some degree from ours which might be expected when considering the inherent differences in cohort composition where ours consisted only of transplanted patients while Walter et al. did not have access to transplant data. The divergence in cohort composition and consequently results is also reflected in the difference in patient age noted by our groups, namely in our analysis M6/M7 patients were older compared with other $\mathrm{FAB}$ types while in the Walter analysis it was the $\mathrm{MO}$ patients who were oldest. Similar to what we noted, their data regarding M7 were also suggestive of inferior clinical outcome, although given the small number of M7 patients ( $0.1 \%$ of the total cohort) they were unable to draw firm conclusions regarding the prognostic role of FAB M7.

In addition to the different study populations (transplanted vs. unknown) analyzed, the difference in the results of both studies may be also accounted by acknowledgment of the different complementary molecular data used, ie, FLT3-ITD compared with CEBPA testing.

An additional salient point we establish is that in FLT3-ITD patients, consideration of FAB type augments patient risk stratification by identifying FAB M2 patients as those with increased relapse incidence, as well as recognizing that M2 and M4 patients as those with decreased leukemia free survival rates. This is of considerable value as most clinicians today would consider allogeneic stem cell transplant the preferred therapeutic approach for fit FLT3-ITD ${ }^{+}$patients, ${ }^{27,28}$ yet even within the patient population positive for FLT3-ITD, relapse risk varies, further emphasizing the need to carefully select patients for whom transplant would be beneficial in terms of the risk to benefit ratio. Recently published data also aiming to stratify FLT3-ITD patients being transplanted indicates that patients with a low FLT3-ITD mutation burden (quantified by the FLT3-ITD mutant to wild-type allelic ratio) do not derive a clinical benefit from an ASCT in terms of RFS and OS. ${ }^{29} \mathrm{How}$ ever, this assay may be limited by low sensitivity, heterogeneous methodologies, and diverse cutoff values used by different centers. ${ }^{27}$ Additionally, our data may better inform clinicians in selecting higher risk patients who may benefit from enrollment into ongoing and future clinical trials with FLT3 targeted maintenance therapy post-transplant.

Given the inherent limitation of a retrospective analysis it may be difficult to determine why FAB M6/M7 patients have decreased leukemia free survival and increased nonrelapse mortality, yet considering that M6 is commonly associated with poor-risk cytogenetics may provide a partial explanation for this clinical phenotype ${ }^{30}$ as well as recognizing that FAB $M 7$ has the highest frequency of cytogenetic aberrations among all FAB categories. ${ }^{31}$ Nevertheless, we do acknowledge that these would not fully explain the increased non-relapse mortality rates observed in our study.

We recognize several limitations in our analysis. First, the retrospective nature of this study with the inherent limitations seen in comparable analyses. Second, our registry is not fully annotated for CEBPA thus limiting our ability to fully characterize the role of FAB classification per the recently revised $\mathrm{WHO}$ classification. Lastly, the relative infrequency of FAB M6/M7 in general and specifically in our analyzed cohort may also limit interpretation of our data.

In aggregate, our findings in a large cohort of transplanted patients suggest that $F A B$ classification contributes and enhances the prognostic capacity of molecular data provided by FLT3-ITD and NPM1 mutational status. Our findings support the continued use of $F A B$ classification to complement molecular data in AML patients undergoing allogeneic stem cell transplantation.

\section{REFERENCES}

[1] Jagasia M, Arora M, Flowers ME, et al. Risk factors for acute GVHD and survival after hematopoietic cell transplantation. Blood 2012; 119:296-307.

[2] Gale RE, Hills R, Kottaridis PD, et al. No evidence that FLT3 status should be considered as an indicator for transplantation in acute myeloid leukemia (AML): an analysis of 1135 patients, excluding acute promyelocytic leukemia, from the UK MRC AML10 and 12 trials. Blood 2005;106:3658-3665.

[3] Pratcorona M, Brunet S, Nomdedeu J, et al. Favorable outcome of patients with acute myeloid leukemia harboring a low-allelic burden FLT3-ITD mutation and concomitant NPM1 mutation: relevance to post-remission therapy. Blood 2013;121:2734-2738.

[4] Bornhauser M, Illmer T, Schaich M, et al. Improved outcome after stem-cell transplantation in FLT3/ITD-positive AML. Blood 2007; 109:2264-2265; author reply 2265. 
[5] Meshinchi S, Arceci RJ, Sanders JE, et al. Role of allogeneic stem cell transplantation in FLT3/ITD-positive AML. Blood 2006;108:400; author reply 400-401.

[6] Brunet S, Labopin M, Esteve J, et al. Impact of FLT3 internal tandem duplication on the outcome of related and unrelated hematopoietic transplantation for adult acute myeloid leukemia in first remission: a retrospective analysis. J Clin Oncol. 2012;30:735-741.

[7] Schmid C, Labopin M, Socie G, et al. Outcome of patients with distinct molecular genotypes and cytogenetically normal $\mathrm{AML}$ after allogeneic transplantation. Blood 2015;126:2062-2069.

[8] Song Y, Magenau J, Li Y, et al. FLT3 mutational status is an independent risk factor for adverse outcomes after allogeneic transplantation in AML. Bone Marrow Transplant 2016;51:511-520.

[9] Rollig C, Bornhauser M, Kramer M, et al. Allogeneic stem-cell transplantation in patients with NPM1-mutated acute myeloid leukemia: results from a prospective donor versus no-donor analysis of patients after upfront HLA typing within the SAL-AML 2003 trial. J Clin Oncol. 2015;33:403-410.

[10] Rollig C, Bornhauser M, Thiede C, et al. Long-term prognosis of acute myeloid leukemia according to the new genetic risk classification of the European LeukemiaNet recommendations: evaluation of the proposed reporting system. J Clin Oncol. 2011;29:2758-2765.

[11] Pfeiffer T, Schleuning M, Mayer J, et al. Influence of molecular subgroups on outcome of acute myeloid leukemia with normal karyotype in 141 patients undergoing salvage allogeneic stem cell transplantation in primary induction failure or beyond first relapse. Haematologica 2013;98:518-525.

[12] Arber DA, Orazi A, Hasserjian R, et al. The 2016 revision to the World Health Organization classification of myeloid neoplasms and acute leukemia. Blood 2016;127:2391-2405.

[13] Chang M, Raimondi SC, Ravindranath Y, et al. Prognostic factors in children and adolescents with acute myeloid leukemia (excluding children with Down syndrome and acute promyelocytic leukemia): univariate and recursive partitioning analysis of patients treated on Pediatric Oncology Group (POG) Study 8821. Leukemia 2000;14:1201-1207.

[14] Jourdan E, Boiron JM, Dastugue N, et al. Early allogeneic stem-cell transplantation for young adults with acute myeloblastic leukemia in first complete remission: an intent-to-treat long-term analysis of the BGMT experience. J Clin Oncol. 2005;23:7676-7684.

[15] Bostrom B, Brunning RD, McGlave P, et al. Bone marrow transplantation for acute nonlymphocytic leukemia in first remission: analysis of prognostic factors. Blood 1985;65:1191-1196.

[16] Zwaan FE, Hermans J, Barrett AJ, et al. Bone marrow transplantation for acute nonlymphoblastic leukaemia: a survey of the European Group for Bone Marrow Transplantation (E.G.B.M.T.). Br J Haematol. 1984;56:645-653.

[17] Fagioli F, Bacigalupo A, Frassoni F, et al. Allogeneic bone marrow transplantation for acute myeloid leukemia in first complete remission: the effect of FAB classification and GVHD prophylaxis. Bone Marrow Transplant 1994;13:247-252.

[18] Walter RB, Othus M, Burnett AK, et al. Significance of FAB subclassification of "acute myeloid leukemia, NOS" in the 2008 WHO classification: analysis of 5848 newly diagnosed patients. Blood 2013;121:2424-2431.

[19] Dohner H, Estey EH, Amadori S, et al. Diagnosis and management of acute myeloid leukemia in adults: recommendations from an international expert panel, on behalf of the European LeukemiaNet. Blood 2010;115:453-474.
[20] Bennett JM, Catovsky D, Daniel MT, et al. Proposals for the classification of the acute leukaemias. French-American-British (FAB) cooperative group. Br J Haematol. 1976;33:451-458.

[21] Amadori S, Venditti A, Del Poeta G, et al. Minimally differentiated acute myeloid leukemia (AML-MO): a distinct clinico-biologic entity with poor prognosis. Ann Hematol. 1996;72:208-215.

[22] Colita A, Belhabri A, Chelghoum Y, et al. Prognostic factors and treatment effects on survival in acute myeloid leukemia of M6 subtype: a retrospective study of 54 cases. Ann Oncol. 2001;12:451-455.

[23] Bennett JM, Catovsky D, Daniel MT, et al. Proposed revised criteria for the classification of acute myeloid leukemia. A report of the French-AmericanBritish Cooperative Group. Ann Intern Med. 1985;103:620-625.

[24] Bennett JM, Catovsky D, Daniel MT, et al. Proposal for the recognition of minimally differentiated acute myeloid leukaemia (AML-MO). Br J Haematol. 1991;78:325-329.

[25] Keating S, Suciu S, de Witte T, et al. Prognostic factors of patients with acute myeloid leukemia (AML) allografted in first complete remission: an analysis of the EORTC-GIMEMA AML 8A trial. The European Organization for Research and Treatment of Cancer (EORTC) and the Gruppo Italiano Malattie Ematologiche Maligne dell' Adulto (GIMEMA) Leukemia Cooperative Groups. Bone Marrow Transplant 1996;17:993-1001.

[26] Tallman MS, Kim HT, Paietta E, et al. Acute monocytic leukemia (FrenchAmerican-British classification M5) does not have a worse prognosis than other subtypes of acute myeloid leukemia: a report from the Eastern Cooperative Oncology Group. J Clin Oncol. 2004;22:1276-1286.

[27] Levis M. FLT3 mutations in acute myeloid leukemia: what is the best approach in 2013? Hematology Am Soc Hematol Educ Program. 2013;2013:220-226.

[28] Brunet S, Martino R, Sierra J. Hematopoietic transplantation for acute myeloid leukemia with internal tandem duplication of FLT3 gene (FLT3/ITD). Curr Opin Oncol. 2013;25:195-204.

[29] Schlenk RF, Kayser S, Bullinger L, et al. Differential impact of allelic ratio and insertion site in FLT3-ITD-positive AML with respect to allogeneic transplantation. Blood 2014:124:3441-3449.

[30] Santos FP, Faderl S, Garcia-Manero G, et al. Adult acute erythroleukemia: an analysis of 91 patients treated at a single institution. Leukemia 2009;23:2275-2280.

[31] Bacher U, Kern W, Schnittger S, et al. Further correlations of morphology according to $\mathrm{FAB}$ and $\mathrm{WHO}$ classification to cytogenetics in de novo acute myeloid leukemia: a study on 2,235 patients. Ann Hematol. 2005;84:785-791.

\section{SUPPORTING INFORMATION}

Additional Supporting Information may be found in the online version of this article.

How to cite this article: Canaani J, Beohou E, Labopin M, et al. Impact of $F A B$ classification on predicting outcome in acute myeloid leukemia, not otherwise specified, patients undergoing allogeneic stem cell transplantation in CR1: An analysis of 1690 patients from the acute leukemia working party of EBMT. Am J Hematol. 2017;92:344-350. https://doi.org/10.1002/ajh.24640 